\title{
Classification of Small-Spot Direct UV Written Fiber Bragg Gratings Through Extreme Thermal Treatment
}

\author{
S.L. Scholl ${ }^{*}$, A. Jantzen ${ }^{1}$, R.H.S. Bannerman ${ }^{1}$, J. Field ${ }^{1}$, J.C. Gates ${ }^{1}$, L.J. Boyd ${ }^{2}$, P.G.R. Smith ${ }^{1}$, C. Holmes ${ }^{1}$ \\ ${ }^{1}$ Optoelectronics Research Centre, University of Southampton, Southampton, SO17 1BJ, UK \\ ${ }^{2}$ Parker Aerospace, Parker Hannifin Corporation, 2510 The Quadrant, Bristol, BS32 4AQ, UK \\ *S.Scholl@soton.ac.uk
}

\begin{abstract}
The high intensity and continuous nature of small-spot direct UV writing makes characterisation of gratings types difficult by conventional approaches. Investigating the thermal response of gratings up to $850{ }^{\circ} \mathrm{C}$ allows elucidation of grating type.

OCIS codes: (060.3735) Fiber Bragg gratings; (060.3738) Fiber Bragg gratings, photosensitivity; (060.2310) Fiber optics
\end{abstract}

\section{Introduction}

Fiber Bragg gratings (FBGs) offer harsh environment sensing capability to a variety of sectors especially aerospace. They specifically offer advantages that include being lightweight, having a small physical footprint, immunity to electromagnetic interference and the ability to withstand high temperatures. This work forms part of a collaboration with Parker Aerospace and reports on high temperature processing of small-spot direct UV written FBGs.

Small-spot direct UV writing (DUW) is a unique fabrication approach, which modulates a focused interference pattern along an optical fiber length. The focused spot is typically $8 \mu \mathrm{m}$ in diameter, leading to high optical intensities and results in a grating written in a single pass. With phase mask writing it is conventional to monitoring grating type formation by observing how the centre wavelength and the grating reflectivity alter during increased exposure of a whole grating [1]. This approach is not well suited to small-spot writing because the grating is written in a single continuous pass, and does not lend itself to observation during writing. Thus it is difficult to classify gratings during the laser writing phase, and so in this work we show that analysis of the thermal response of individual gratings, written at different fluences, can be used to obtain a better understanding of the writing mechanisms. This approach builds on that described by Canning [1], whereby different types of gratings can be categorised by their thermal properties.

The work also identifies thermal regeneration and parameters that extend the operational range of the gratings. This expands on previous work made by the group [2] and potentially permits harsh environment aerospace deployments to be realized.

\section{Fabrication}

DUW-FBGs are written with an interference pattern created by separating, focusing and recombining two coherent laser beams to form an $8 \mu \mathrm{m}$ spot in the fiber core as shown in Fig. 1. The fiber core is aligned and translated through the focused beam and gratings are defined through synchronized phase modulation of arms.

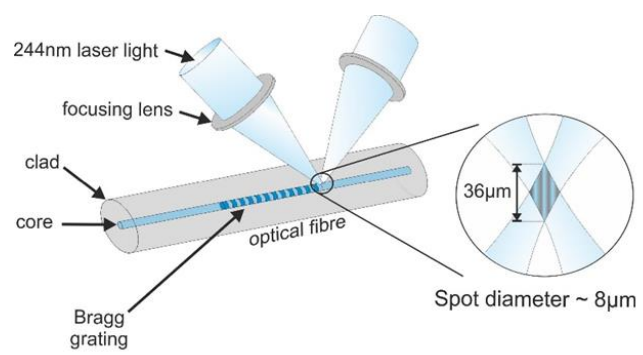

Fig. 1 Schematic drawing of the small-spot DUW fiber Bragg grating writing process, creating an interference pattern in a spot with approximately $8 \mu \mathrm{m}$ diameter [3]

The reported technique uses a $244 \mathrm{~nm}$ argon ion UV continuous wave laser, it is split into two coherent paths, which are focused and combined to an $8 \mu \mathrm{m}$ diameter spot in the core of the fiber. The interference pattern formed in the fiber core establishes a periodic refractive index change of the photosensitive core material. An electro-optic modulator (EOM) is inserted into the path of one beam for phase control during spot translation. The translation 
speed of the fiber with respect to the focused beam determines the exposure. Through computer controlled synchronized modulation, apodisation and detuning can be implemented into the grating design.

To analyze the thermal behavior, eleven $5 \mathrm{~mm}$ long gratings were written into a GF4A optical fiber core in a wavelength range from $1502 \mathrm{~nm}$ to $1522 \mathrm{~nm}$ with a spectral separation of $2 \mathrm{~nm}$. Each Bragg grating was written with a different nominal fluence from 10 to $40 \mathrm{~kJ} / \mathrm{cm}^{2}$. The normalized reflection strength can be seen in Fig. 2 (a).

Thermal treatment of the fiber was performed in a tube furnace using an argon environment. The spectral reflection was recorded during the thermal treatment by connecting the fiber via a 50:50 coupler to a broadband light source and an optical spectrum analyzer. The temperature was ramped up at $5{ }^{\circ} \mathrm{C} / \mathrm{min}$ from room temperature to $850{ }^{\circ} \mathrm{C}$, where the temperature dwelled for 30 minutes and subsequently ramped down at $5{ }^{\circ} \mathrm{C} / \mathrm{min}$ to room temperature.

\section{Results}

The reflectance spectrum during thermal treatment was recorded in 22 second intervals along with a reference temperature reading.

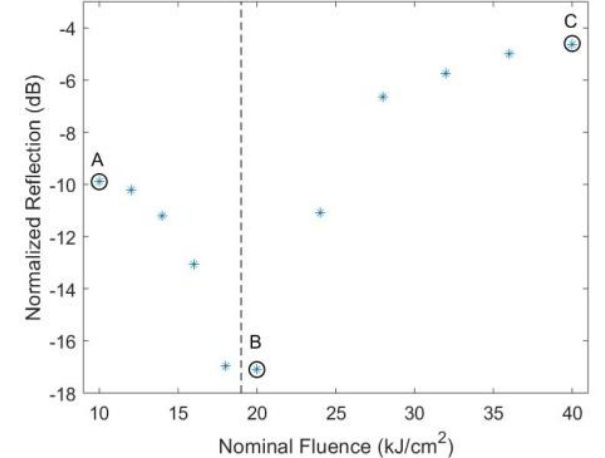

(a)

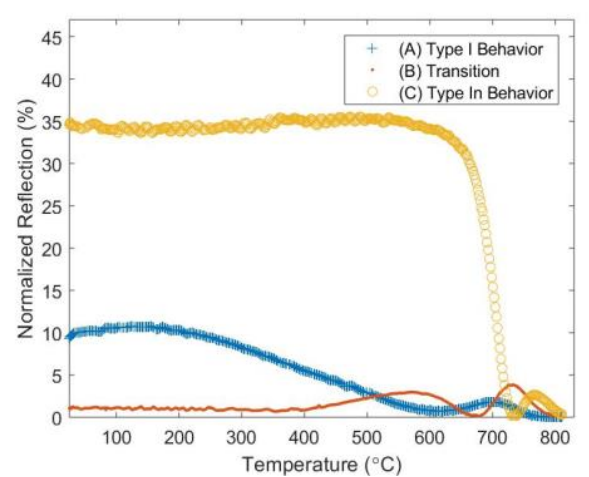

(b)

Fig. 2 (a) Normalized reflection over nominal fluence for 11 gratings in GF4A at room temperature. The thermal behavior of gratings A, B, C are displayed in Fig. 2b. (b) The normalized reflection of three gratings written at different fluences is plotted during thermal treatment. The gratings demonstrate different thermal behavior, which enables an allocation into the grating types Type I, Type In behavior and a transitional grating.

The normalized reflection over the nominal fluence at room temperature is shown in Fig. 2 (a). With an increase of nominal fluence, the grating strength dips before raising, which indicates a transition of grating types. To confirm the initial assumption, the normalized reflection over the complete temperature range was captured for fluence A, B and $\mathrm{C}$ that are shown in Fig. 2 (a) and their thermal profiles are given in Fig. 2 (b). The grating, which was written with a nominal fluence of $10 \mathrm{~kJ} / \mathrm{cm}^{2}$ starts degrading at $200^{\circ} \mathrm{C}$, reaching its lowest reflection at $600{ }^{\circ} \mathrm{C}$, before it regenerates and finally anneals out at $800{ }^{\circ} \mathrm{C}$. This thermal behavior is characteristic for Type I gratings. The grating that is temperature stable until $650{ }^{\circ} \mathrm{C}$, where it abruptly degrades, regenerates and anneals out at $810^{\circ} \mathrm{C}$, was written with a fluence of $40 \mathrm{~kJ} / \mathrm{cm}^{2}$. The high temperature stability indicates a Type In behavior [1]. The intermediate grating, written with $20 \mathrm{~kJ} / \mathrm{cm}^{2}$ has a thermal stability of a Type I grating with degrading at lower temperatures, whilst strongly regenerating at higher temperatures and hence demonstrating Type In behavior. The thermal regeneration varies in strength and thermal position for each grating type.

\section{Conclusion}

This work presents a method for categorizing the grating type for small-spot direct UV written Bragg gratings. The work quantifies the thermal response and thermal regeneration in GF4A. The presented work shall be extended to consider the spectral behavior of other types of optical fiber including SMF-28.

\section{References}

[1] J. Canning, "Fibre gratings and devices for sensors and lasers", Laser \& Photon. Rev., No. 4, 275-289 (2008).

[2] A. Jantzen, R. Bannerman, S. Berry, J. Gates, P. Gow, L. Boyd, P. Smith, and C. Holmes, "Observations from direct UV-written, nonhydrogen-loaded, thermally regenerated Bragg gratings in double-clad photosensitive fiber," Opt. Lett. 42, 3741-3744 (2017).

[3] R. Bannerman, M. Posner, C. Holmes, P. Mennea, P. Smith, and J. Gates, "Insertion Loss Characterisation for UV Written Bragg Gratings in Fiber and Planar Waveguides," in 2017 European Conference on Lasers and Electro-Optics and European Quantum Electronics Conference, (Optical Society of America, 2017), paper CE_P_27. 